\title{
High precision determination of the $D$ and Ds decay constants and masses from lattice QCD
}

\section{Eduardo Follana* and C. T. H. Davies}

University of Glasgow, Glasgow, $U K$

\section{G. P. Lepage}

Cornell University, Ithaca, New York, USA

\section{J. Shigemitsu}

The Ohio State University, Columbus, Ohio, USA

\section{(HPQCD collaboration)}

\begin{abstract}
We present a determination of the decay constants of the $D$ and $D_{s}$ mesons from lattice QCD, each with a total error of about $2 \%$, approximately a factor of three better than previous calculations. We have been able to achieve this through the use of a highly improved discretization of QCD for charm quarks, coupled to gauge configurations generated by the MILC collaboration that include the full effect of sea $u, d$, and $s$ quarks. We have results for a range of $u / d$ masses down to ms/5 and three values of the lattice spacing, which allow us to perform accurate continuum and chiral extrapolations. We fix the charm quark mass to give the experimental value of the $\eta_{c}$ mass, and then a stringent test of our approach is the fact that we obtain correct (and accurate) values for the mass of the $D$ and $D s$ mesons. We compare $f_{D}$ and $f_{D_{s}}$ with $f_{K}$ and $f_{\pi}$, and using experiment determine corresponding CKM elements with good precision.
\end{abstract}

The XXV International Symposium on Lattice Field Theory

July 30 - August 42007

Regensburg, Germany

* Speaker. 


\section{Introduction}

Precision calculations in lattice QCD play a crucial role in testing our non-perturbative theoretical tools, by comparing the results of the calculation with precisely measured quantities. On the other hand accurate calculations of non-perturbative QCD quantities are very important in the analysis of experimental data, for example in the determination of the Cabibbo-Kobayashi-Maskawa (CKM) matrix elements.

This is most clearly seen in the case of "gold-plated" processes, for example the leptonic decay of $D_{s}, D_{d}, \pi$ and $K$ mesons. In this process the corresponding meson, with quark content $a \bar{b}$ (or $\bar{a} b$ ) annihilates weakly into a $\mathrm{W}$ boson, with a width given, up to calculated electromagnetic corrections $[1,2]$, by:

$$
\Gamma\left(P \rightarrow l v_{l}(\gamma)\right)=\frac{G_{F}^{2}\left|V_{a b}\right|^{2}}{8 \pi} f_{P}^{2} m_{l}^{2} m_{P}\left(1-\frac{m_{l}^{2}}{m_{P}^{2}}\right)^{2} .
$$

$V_{a b}$ is the corresponding element of the CKM matrix, and the decay constant $f_{P}$ parametrises the amplitude for $\mathrm{W}$ annihilation. By combining a measurement of $\Gamma$ with an accurate calculation of $f_{P}$ (1.1) can be used to determine $V_{a b}$. If $V_{a b}$ is known from elsewhere we can use (1.1) to get a value for $f_{P}$.

The decay constant $f_{P}$ is conventionally defined to be a property of the pseudoscalar meson, calculable in QCD without QED effects, and is given by:

$$
\left\langle 0\left|\bar{a} \gamma_{\mu} \gamma_{5} b\right| P(p)\right\rangle=f_{P} p_{\mu} .
$$

The calculation of $f_{P}$ is a hard non-perturbative problem, which at present can only be done fully with lattice QCD. There are very precise experimental measurements for the leptonic decay rates in the case of the $\pi$ and $K$, and new results are appearing for $D$ and $D_{s}$, which make the calculations a highly non-trivial test of lattice QCD, and ultimately of QCD itself. This tests are important to give us confidence in similar lattice QCD calculations of matrix elements in B systems, for which experimental results are much harder to obtain.

\section{Improved Staggered Quarks}

We use HISQ staggered quarks in the valence sector, whereas the sea quarks are ASQTAD staggered quarks with the fourth root trick $[3,4,5]$.

The massless one-link (Kogut-Susskind) staggered Dirac operator is defined as:

$$
D(x, y)=\frac{1}{2 a u_{0}} \sum_{\mu=1}^{d} \eta_{\mu}(x)\left[U_{\mu}(x) \delta_{x+\hat{\mu}, y}-H . c .\right], \quad \eta_{v}(x)=(-1)^{\sum_{\mu<v} x_{\mu}}
$$

with $u_{0}$ an optional tadpole-improvement factor. This operator suffers from doubling: there are four "tastes" (non-physical flavours) of fermions in the spectrum, which couple through taste-changing interactions. These are lattice artifacts of order $a^{2}$, involving at leading order the exchange of a gluon of momentum $q \approx \pi / a$. Such interactions are perturbative for typical values of the lattice spacing, and can be corrected systematically a la Symanzik. By judiciously smearing the gauge field we can remove the coupling between quarks and high momentum gluons. 
The most widely used improved staggered action is called ASQTAD, and removes all treelevel $a^{2}$ discretization errors in the action $[6,7,8]$.

The HISQ (highly improved staggered quarks) staggered Dirac operator involves two levels of smearing with an intermediate projection onto $S U(3)$. It is designed so that, as well as eliminating all tree-level $a^{2}$ discretization errors, it further reduces the one-loop taste-changing errors (see [9] for a more detailed discussion.) This action has been shown to substantially reduce the errors associated with the taste-changing interactions $[9,10,11]$.

When we put massive quarks on the lattice, the discretization errors grow with the quark mass as powers of $a m$. Therefore to obtain small errors we would need $a m \ll 1$. For heavy quarks this would require very small lattice spacings. On the other hand, to keep our lattice big enough to accommodate the light degrees of freedom, we need $L a \gg m_{\pi}^{-1}$. The fact that we have two very different scales in the problem makes difficult a direct solution. What we can do instead is to take advantage of the fact that $m$ is large, by using an effective field theory (NRQCD, HQET). This program has been very successful for b quarks $[12,13,14]$.

The charm quark is in between the light and heavy mass regime. It is quite light for an easy application of NRQCD, but quite large for the usual relativistic quark actions, $a m_{c} \lesssim 1$. However, if we use a very accurate action (HISQ) and fine enough lattices (fine MILC ensembles), it is possible to get results accurate at the few percent level. A non-relativistic analysis [9] shows that for HISQ charm quarks the largest remaining source of error is due to the quark's energy, and can be further suppressed by powers of $v / c$, where $v$ is the typical velocity of the quark in the system of interest, simply by retuning the overall coefficient of the Naik term to impose the correct relativistic dispersion relation $c^{2}(p)=1$ for low lattice momentum $p$.

One advantage of the use of a relativistic action is the existence of a partially conserved current, which implies the non-renormalisation of the lattice result for $f_{P}$. We can extract $f_{P}$ from the PCAC relation for zero momentum meson $\mathrm{P}$ :

$$
f_{P} m_{P}^{2}=\left(m_{a}+m_{b}\right)\left\langle 0\left|\bar{a} \gamma_{5} b\right| P\right\rangle
$$

\section{Results}

We use $2+1$-flavours unquenched configurations generated by the MILC collaboration [15, 16, 17]. The parameters of the ensembles we have used for both the sea and the valence sectors are in table 1 . The lattice results are converted to physical units through the heavy quark potential parameter $r_{1}$, as determined by the MILC collaboration (table 1, [16]). The physical value of $r_{1}$ is determined from the $\Upsilon$ spectrum calculated in NRQCD with $b$ quarks on the same MILC ensembles [13], with the result $r_{1}=0.321(5) \mathrm{fm}, r_{1}^{-1}=0.615(10) \mathrm{GeV}$.

We use multiple precessing random wall sources, which gives a 3-4-fold reduction in statistical errors with respect to local sources.

The mass of the charm quark is fixed by adjusting the mass of the "goldstone " $\eta_{c}$ to its experimental value. The light $(\mathrm{u} / \mathrm{d})$ and strange quark masses are fixed using the experimental values for the masses of $\pi$ and $K$. Our results use masses for the light quarks that are substantially larger (by a factor of around three) than the real ones. In order to get physical answers we extrapolate to the correct $u / d$ mass using chiral perturbation theory. Once the masses have been thus fixed, there 


\begin{tabular}{l|l|l}
$\begin{array}{l}\text { Lattice/sea } \\
u_{0} a m_{l}, u_{0} a m_{s}\end{array}$ & $\begin{array}{l}\text { valence } \\
a m_{l}, \quad a m_{s}, \quad a m_{c}, 1+\varepsilon\end{array}$ & $r_{1} / a$ \\
\hline $16^{3} \times 48$ & & \\
$0.0194,0.0484$ & $0.0264,0.066,0.85,0.66$ & $2.129(11)$ \\
$0.0097,0.0484$ & $0.0132,0.066,0.85,0.66$ & $2.133(11)$ \\
$20^{3} \times 64$ & & \\
$0.02,0.05$ & $0.0278,0.0525,0.648,0.79$ & $2.650(8)$ \\
$0.01,0.05$ & $0.01365,0.0546,0.66,0.79$ & $2.610(12)$ \\
$24^{3} \times 64$ & & \\
$0.005,0.05$ & $0.0067,0.0537,0.65,0.79$ & $2.632(13)$ \\
$28^{3} \times 96$ & & \\
$0.0124,0.031$ & $0.01635,0.03635,0.427,0.885$ & $3.711(13)$ \\
$0.0062,0.031$ & $0.00705,0.0366,0.43,0.885$ & $3.684(12)$
\end{tabular}

Table 1: MILC configurations and mass parameters used for this analysis. The $16^{3} \times 48$ lattices are 'very coarse', the $20^{3} \times 64$ and the $24^{3} \times 64$, 'coarse' and the $28^{3} \times 96$, 'fine'. The sea ASQTAD quark masses $(l=u / d)$ are given in the MILC convention where $u_{0}$ is the plaquette tadpole parameter. Note that the sea $s$ quark masses on fine and coarse lattices are above the subsequently determined physical value [17]. The lattice spacing values in units of $r_{1}$ after 'smoothing' are given in the rightmost column [16, 18]. The third column gives the HISQ valence $u / d, s$ and $c$ masses along with the coefficient of the Naik term, $1+\varepsilon$, used for $c$ quarks [9].

is no remaining freedom to change any parameters, and in particular the results we obtain for the masses of heavy-light mesons are a stringent test of our method.

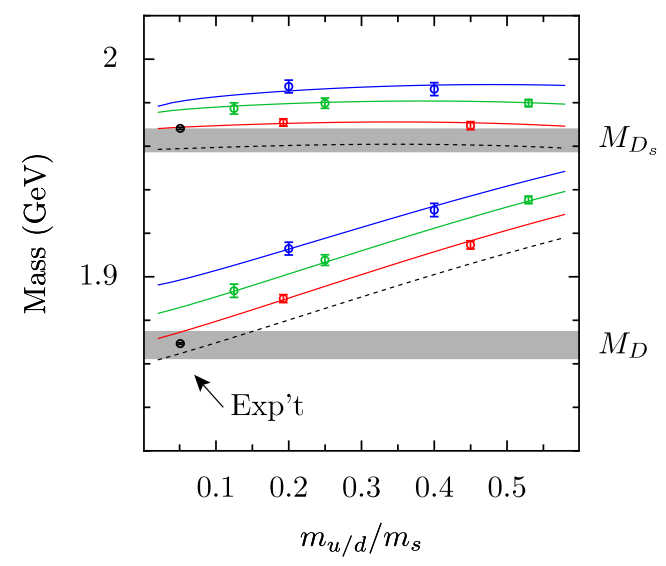

Figure 1: Masses of the $D^{+}$and $D_{s}$ meson. The lines give the simultaneous chiral fits and the dashed line the continuum extrapolation. Our final error bars are given by the shaded bands. These are offset from the dashed lines by an estimate of electromagnetic, $m_{u} \neq m_{d}$ and other systematic corrections to the masses. The experimental results are marked at the physical $m_{d} / m_{s}$.



Figure 2: $\quad$ Results for the $D, D_{s}, K$ and $\pi$ decay constants. Symbols as in the previous figure. At the left are experimental results from CLEO-c [22, 24] and BaBar [23] ( $D_{s}$ only) and from the Particle Data Tables [2] for $K$ and $\pi$. 
In addition to the chiral extrapolation, we have systematic errors coming from a variety of sources [19], among them from the finite lattice spacing. Because we have three different lattice spacings and very precise data, we can extrapolate to the continuum limit. This extrapolation is linked to the chiral extrapolation through discretization errors in the light quark action. We therefore perform a simultaneous bayesian fit for both chiral and continuum extrapolations, allowing for a range of expected functional forms in both. We tested the validity of the method by fitting hundreds of fake datasets generated using staggered chiral perturbation theory with random couplings. We fit simultaneously to the masses and the decay constants, that is, we fit $m_{\pi}, m_{K}, f_{\pi}$ and $f_{K}$ simultaneously, and similarly for $m_{D}, m_{D_{s}}, f_{D}$ and $f_{D_{s}}$. We present some of the results in figures 1 and 2.

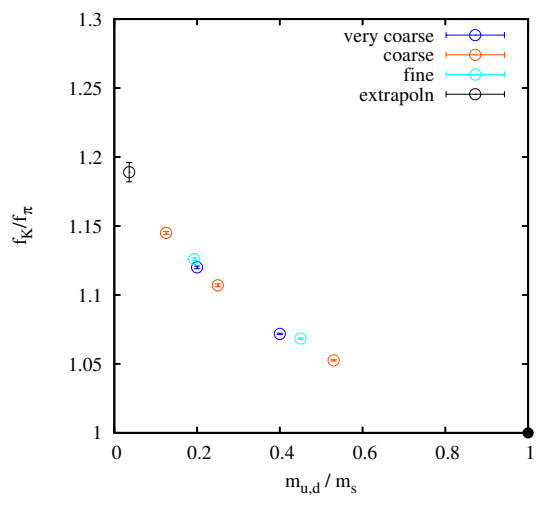

Figure 3: Ratio of decay constants $f_{K} / f_{\pi}$ on very coarse, coarse and fine ensembles, as a function of the $u, d$ quark mass in units of the $s$ quark mass.

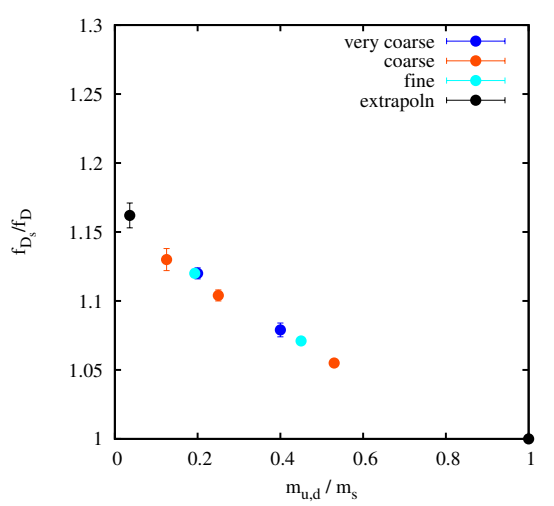

Figure 4: Ratio of heavy-light decay constants $f_{D_{s}} / f_{D}$ on very coarse, coarse and fine ensembles, as a function of the $u, d$ quark mass in units of the $s$ quark mass.

We get an excellent agreement with experiment for the masses: $m_{D_{s}}=1.963(5) \mathrm{GeV}$ (experiment $1.968 \mathrm{GeV}$ ), and $m_{D}=1.869(6) \mathrm{GeV}$ (experiment $1.869 \mathrm{GeV}$ ). Our calculation also reproduces correctly the difference in binding energies between a heavy-heavy $\left(\eta_{c}\right)$ and a heavy-light $\left(m_{D}\right.$ and $\left.m_{D_{s}}\right)$ state: $\left(2 m_{D_{s}}-m_{\eta_{c}}\right) /\left(2 m_{D}-m_{\eta_{c}}\right)=1.249(14)$ (experiment $\left.1.260(2)\right)$. This has not been possible with charm quark calculations in lattice QCD before.

We also have agreement with experiment for the light-light decay constants [19]. The result for the ratio is very accurate, $f_{K} / f_{\pi}=1.189(7)$, and shows tiny discretization effects (figure 3). Combining this ratio with experimental leptonic branching fractions [17, 20] we get $V_{u s}=0.2262(13)(4)$, where the first error is theoretical and the second experimental. This gives the unitarity relation $1-V_{u d}^{2}-V_{u s}^{2}-V_{u b}^{2}=0.0006(8)$.

Our results for the heavy-light decay constants are 4-5 times more accurate than previous lattice QCD results and existing experimental measurements: $f_{D_{s}}=241(3) \mathrm{MeV}, f_{D}=208(4)$ $\mathrm{MeV}$, and a ratio of $f_{D_{s}} / f_{D}=1.162(9)$ (see figure 4). For the double ratio $\left(f_{D_{s}} / f_{D}\right) /\left(f_{K} / f_{\pi}\right)$, which is estimated to be close to 1 from low order chiral perturbation theory [21], we get a value of $0.977(10)$.

The experimental leptonic branching rates, together with CKM matrix elements determined from other processes (assuming $V_{c s}=V_{u d}$ ) give a value for $f_{D_{s}}$ of 264(17) MeV for $\mu$ decays 
and 310(26) MeV for $\tau$ decay from CLEO-c [22] and 283(23) MeV from BaBar [23], and for $f_{D} 223(17) \mathrm{MeV}$ from CLEO-c for $\mu$ decay [24]. Using our results for $f_{D_{s}}$ and $f_{D_{s}} / f_{D}$ and the experimental values from CLEO-c [22] for $\mu$ decay (since the electromagnetic corrections are wellknown in that case) we can directly determine the corresponding CKM elements: $V_{c s}=1.07(1)(7)$ and $V_{c s} / V_{c d}=4.42(4)(41)$. The first error is theoretical and the second experimental. The result for $V_{c s}$ improves on the direct determination of 0.96(9) given in the Particle Data Tables [2].

\section{Conclusions and outlook}

We have shown that the use of a highly improved relativistic action on fine enough lattices is capable of delivering very precise results on systems with a charm quark. The high statistical accuracy of our data combined with calculations at several values of the lattice spacing and light quark masses allows us to make a controlled joint chiral and continuum extrapolation.

We can calculate accurately the mass of heavy-light systems, which provide a stringent test of the calculation. We can calculate precise values for the decay constants of pseudoscalar heavy-light mesons (as well as light-light mesons), and especially for the ratio of such decay constants.

The very precise calculation of the masses of heavy-heavy pseudoscalar mesons should make possible a direct lattice determination of the mass of the charm quark. Because we use the same relativistic action through the calculation for both the charm and the light quarks, we can also obtain a very precise value for the ratio $m_{c} / m_{s}$, and therefore if $m_{c}$ is determined through another method use the ratio to get $m_{s}$.

Another quantity which we plan to calculate in the near future is the leptonic decay width $\Gamma_{e^{+} e^{-}}(\psi)$, as well as the semileptonic form factors for $D \rightarrow \pi l v, D \rightarrow K l v$.

\section{Acknowledgments}

We are grateful to the MILC collaboration for the use of their configurations and to Quentin Mason and Doug Toussaint for useful discussions. The computing was done on Scotgrid and the QCDOCX cluster. This work was supported by PPARC, the Royal Society, NSF and DoE.

\section{References}

[1] W. J. Marciano and A. Sirlin, Phys. Rev. Lett. 71, 3629 (1993).

[2] W. M. Yao et al, J. Phys. G33, 1 (2006).

[3] S. Sharpe, PoS LAT2006:022 (2006) [hep-lat/0610094].

[4] M. Creutz, PoS LAT2007 (2007) [arXiv:0708.1295].

[5] A. Kronfeld, PoS LAT2007 (2007).

[6] S. Naik, Nucl. Phys. B316, 238 (1989).

[7] G. P. Lepage, Phys. Rev. D59, 074502 (1999) [hep-lat/9809157].

[8] K. Orginos, D. Toussaint and R.L. Sugar, Phys. Rev. D60 054503 (1999) [hep-lat/9903032].

[9] E. Follana, Q. Mason, C. T. H. Davies, K. Hornbostel, G. P. Lepage, J. Shigemitsu, H. Trottier and K. Wong, HPQCD and UKQCD collaborations, Phys. Rev. D75:054502, (2007) [hep-lat/0610092]. 
[10] E. Follana, A. Hart, C. T. H. Davies, Phys. Rev. Lett. 93241601 (2004) [hep-lat/0406010].

[11] E. Follana, A. Hart, C. T. H. Davies, Phys. Rev. D72 054501 (2005) [hep-lat/0507011].

[12] A. Gray, M. Wingate, C. T. H. Davies, E. Gulez, G. P. Lepage, Q. Mason, M. Nobes, J. Shigemitsu, Phys.Rev.Lett. 95212001 (2005) [hep-lat/0507015].

[13] A.Gray, I. Allison, C. T. H. Davies, E. Gulez, G. P. Lepage, J. Shigemitsu and M. Wingate, Phys.Rev. D72 094507 (2005) [hep-lat/0507013].

[14] C. Aubin, C. Bernard, C. DeTar, M. Di Pierro, E. D. Freeland, Steven Gottlieb, U. M. Heller, J. E. Hetrick, A. X. El-Khadra, A. S. Kronfeld, L. Levkova, P. B. Mackenzie, D. Menscher, F. Maresca, M. Nobes, M. Okamoto, D. Renner, J. Simone, R. Sugar, D. Toussaint, H. D. Trottier, Phys.Rev.Lett. 95 122002 (2005) [hep-lat/0506030].

[15] C. Bernard, T. Burch, T. A. DeGrand, S. Datta, C. DeTar, S. Gottlieb, U. M. Heller, K. Orginos, R. Sugar, D. Toussaint, Phys.Rev. D64 054506 (2001) [hep-lat/0104002].

[16] C. Aubin, C. Bernard, C. DeTar, S. Gottlieb, E. B. Gregory, U. M. Heller, J. E. Hetrick, J. Osborn, R. Sugar, D. Toussaint, Phys.Rev. D70 (2004) 094505 (2004) [hep-lat/0402030].

[17] C. Aubin, C. Bernard, C. DeTar, Steven Gottlieb, E.B. Gregory, U.M. Heller, J.E. Hetrick, J. Osborn, R. Sugar, D. Toussaint, Phys.Rev. D70 (2004) 114501 [hep-lat/0407028].

[18] Private communication, FNAL/MILC collaborations.

[19] E. Follana, C. T. H. Davies, G. P. Lepage, J. Shigemitsu, [arXiv:0706.1726].

[20] W. J. Marciano, Phys. Rev. Lett. 93:231803 (2004) [ hep-ph/0402299].

[21] D. Becirevic, S. Fajfer, S. Prelovsek, J. Zupan, Phys. Lett. B563:150 (2003) [hep-ph/0211271]

[22] M .Artuso et al, CLEO collaboration, [arXiv:0704.0629]; T. K. Pedlar et al, CLEO collaboration, [arXiv:0704.0437].

[23] B. Aubert et al, BaBar collaboration, Phys. Rev. Lett. 98:141801 (2007) [hep-ex/0607094].

[24] M. Artuso et al, CLEO collaboration, Phys. Rev. Lett. 95:251801 (2005) [hep-ex/0508057]. 